\title{
A Comparison of the X-Ray Properties of FU Ori-type Stars to Generic Young Stellar Objects
}

\author{
Michael A. Kuhn (10) and Lynne A. Hillenbrand \\ Department of Astronomy, California Institute of Technology, Pasadena, CA 91125, USA; mkuhn@astro.caltech.edu \\ Received 2019 May 10; revised 2019 July 31; accepted 2019 August 9; published 2019 September 26
}

\begin{abstract}
Like other young stellar objects (YSOs), FU Ori-type stars have been detected as strong X-ray emitters. However, little is known about how the outbursts of these stars affect their X-ray properties. We assemble available X-ray data from XMM-Newton and Chandra observations of $16 \mathrm{FU}$ Ori stars, including a new XMM-Newton observation of Gaia 17bpi during its optical rise phase. Of these stars, six were detected at least once, while 10 were nondetections, for which we calculate upper limits on intrinsic X-ray luminosity $\left(L_{\mathrm{X}}\right)$ as a function of plasma temperature $(k T)$ and column density $\left(N_{\mathrm{H}}\right)$. The detected FU Ori stars tend to be more X-ray luminous than is typical for non-outbursting YSOs, based on comparison to a sample of low-mass stars in the Orion Nebula Cluster. FU Ori stars with high $L_{\mathrm{X}}$ have been observed both at the onset of their outbursts and decades later. We use the Kaplan-Meier estimator to investigate whether the higher X-ray luminosities for FU Ori stars are characteristic or a result of selection effects, and we find the difference to be statistically significant $(p<0.01)$ even when nondetections are taken into account. The additional X-ray luminosity of FU Ori stars relative to non-outbursting YSOs cannot be explained by accretion shocks, given the high observed plasma temperatures. This suggests that, for many FU Ori stars, either (1) the outburst leads to a restructuring of the magnetosphere in a way that enhances X-ray emission, or (2) FU Ori outbursts are more likely to occur among YSOs with the highest quiescent X-ray luminosity.
\end{abstract}

Key words: accretion, accretion disks - stars: formation - stars: individual (Gaia 17bpi) - stars: variables: T Tauri, Herbig $\mathrm{Ae} / \mathrm{Be}-\mathrm{X}$-rays: stars

\section{Introduction}

An FU Ori-type star is a young stellar object (YSO) in a state of outburst, during which its optical brightness increases by 4-6 mag on a timescale of months to years and declines over a timescale of decades to centuries (Reipurth \& Aspin 2010). These events are caused by a change in structure of the circumstellar disk, resulting in high accretion rates and causing the disk to outshine the central star in the optical and infrared (Hartmann \& Kenyon 1996). However, our understanding of these objects is based on only a handful of sources where the initial outburst was observed, along with a small number of other stars that exhibit FU Ori-like characteristics (see Connelley \& Reipurth 2018).

The changes that produce these outbursts could also be expected to affect the X-ray emission from these YSOs. For ordinary pre-main-sequence stars, $\mathrm{X}$-ray emission is dominated by coronal plasmas with temperatures of tens of millions of degrees Kelvin heated by magnetic reconnection (Feigelson \& Montmerle 1999; Güdel 2004). This mechanism is thought to be similar to the process that heats the corona of main-sequence stars like the Sun, but is scaled up by a factor of $10^{3}-10^{5}$ due to stronger magnetic dynamos in pre-main-sequence stars (Preibisch et al. 2005). Accretion onto YSOs can also produce shock heating. The maximum temperature of gas heated this way is limited to several million Kelvin by the freefall velocity onto the star, yielding a cooler component of X-ray emission that is more easily absorbed by the surrounding material. However, accretion-generated X-ray emission has been observed in some $\mathrm{T}$ Tauri stars with X-ray spectroscopy (Kastner et al. 2002; Schmitt et al. 2005). Outflowing jets are another possible source of X-rays from YSOs. X-ray observations of these jets have typically revealed shock-heated gas with temperatures of several million Kelvin (Bonito et al. 2007).

Several processes in FU Ori stars could either suppress or enhance the X-ray production mechanisms described above. During an outburst, it is thought that the circumstellar disk moves inward toward the star, possibly crushing the stellar magnetosphere (Shu et al. 1994). However, there has been little examination of how this would affect X-ray production. For non-outbursting $\mathrm{T}$ Tauri stars, observations have indicated that accretion suppresses X-ray emission (Telleschi et al. 2007), possibly as the result of mixing between accreted gas and magnetically heated plasma, cooling the hot gas. It also seems likely that changes in the configuration of material in the disk, accretion streams, or outflows could increase the absorption of $\mathrm{X}$-ray emission produced close to the central star. On the other hand, higher accretion rates and stronger jets seem likely to enhance soft X-ray emission.

Our understanding of X-ray emission from FU Ori stars has been based on a small number of studies of individual objects, including FU Ori itself (Skinner et al. 2010), HBC 722 (Liebhart et al. 2014), V960 Mon (Pooley et al. 2015), V1735 Cyg (Skinner et al. 2009), L1551 IRS 5 (Bally et al. 2003; Schneider et al. 2011), and Z CMa (Stelzer et al. 2009). Several of these studies have noted particularly high X-ray luminosities compared to what is expected for typical YSOs (e.g., Skinner et al. 2009, 2010). Furthermore, Liebhart et al. (2014) reported increasing X-ray luminosity of HBC 722 in three observations during the first several years of its outburst. The $\mathrm{X}$-ray emission from most of these objects is dominated by a high-temperature component that is most likely the result of magneticreconnection heating. However, non-detections, which have 
resulted from most X-ray observations, have been largely ignored $^{1}$, which results in a selection bias in the literature that makes it difficult to draw conclusions about the overall population.

In order to better understand possible changes in X-ray properties of FU Ori stars, we acquired an XMM-Newton observation of the most recent FU Ori star, Gaia 17bpi, shortly after its discovery. Gaia $17 \mathrm{bpi}$ is a spectroscopically confirmed FU Ori-type star (Hillenbrand et al. 2018) that triggered a Gaia Science Alert (Hodgkin et al. 2013) in 2017. The source has continued to brighten in the Gaia $G$ band, increasing by 4 mag as of mid-2019.

We pool the available X-ray observations of FU Ori stars, including Gaia 17bpi, to investigate whether there is sufficient evidence to determine if the outbursts affect X-ray emission from these stars. Section 2 describes the available X-ray data for FU Ori stars and our reduction methodology for Gaia 17bpi. In Section 3, we derive constraints on X-ray fluxes and model parameters for non-detected FU Ori stars and compare these to expectations for non-outbursting YSOs. Section 4 examines the properties of the full FU Ori sample, constructing an X-ray luminosity function (XLF) that includes both detections and non-detections and comparing this distribution to the XLF for ordinary YSOs. Finally, Section 5 discusses implications of enhanced X-ray emission observed from some FU Ori stars.

\section{X-Ray Observations and Data Reduction}

\subsection{XMM-Newton Observation of Gaia 17bpi}

Gaia $17 \mathrm{bpi}$ was observed by XMM-Newton at our request as an unanticipated target of opportunity (TOO) after its classification as an FU Ori-type star. The observation (ID 0821400201; PI: Norbert Schartel) was made during XMM-Newton revolution 3468 on 2018 November 15 with a nominal aim-point of 19:31:05.60+18:27:52.0. Observations with EPIC's MOS1 (36,747 s), MOS2 (36,731 s), and pn $(35,203 \mathrm{~s})$ detectors were made in full frame mode using the thin filter. RGS1 and RGS2 were also active during the observation; however, the low flux from Gaia 17bpi means that only the EPIC instrument provides constraints on X-ray flux. The optical monitor (OM) took a series of exposures, rotating sequentially through the $V, U$, and $U V W 1$ filters.

X-ray imaging array data from EPIC were reduced using the XMM-Newton threads with SAS version 17.0.0 (SAS Development Team 2014), HEASOFT version 6.25 (HEASARC 2014), and calibration files from 2018 November 27. We created calibrated event files using the SAS procedures epproc and emproc, removed time intervals with background flaring, and applied a barycentric correction. We retain events from the pn and MOS detectors with PATTERN $<12,0.2<\mathrm{PI}<12 \mathrm{keV}$, and the standard \#XMMEA_EP and \#XMMEA_EM flags.

An X-ray source was not apparent at the location of Gaia 17bpi in the EPIC images. Our analysis focuses on the pn detector due to its greater sensitivity. To determine limits on X-ray flux from Gaia 17bpi, source extraction was performed using a circular aperture at the location of the star (19:31:05.60

\footnotetext{
1 Upper limits have been published for V1057 Cyg and V1515 Cyg by Skinner et al. (2010) and a non-detection in an initial observation of HBC 722 is discussed by Liebhart et al. (2014). Additional detections and/or nondetections are available in archival data sets for those and other FU Ori stars as presented in Section 3.
}

$+18: 27: 52.0$ ICRS) with a radius of $15^{\prime \prime}$. We also defined nine background apertures of the same size on the same chip with distances from the target source ranging from $1^{\prime}$ to $2^{\prime}$, which were selected to avoid other nearby X-ray sources. Observational properties that affect the conversion between detector events and photons, including telescope throughput, detector sensitivity, vignetting, bad pixels, and light lost from the aperture are encoded in the auxiliary response file (ARF) generated by arfgen.

The OM images were reduced by the Processing Pipeline Subsystem (PPS) to yield an ultraviolet/optical catalog. Gaia $17 \mathrm{bpi}$ was detected in the $U$ and $V$ bands, but not in $U V W 1$, by the omdetect algorithm, and the source was extracted using 6 pixel apertures. The photometry in the XMM-Newton OM Vega-magnitude system is $U V W 1>20.4 \mathrm{mag}, \quad U=$ $21.12 \pm 0.23 \mathrm{mag}$, and $V=17.75 \pm 0.04 \mathrm{mag}$.

\subsection{X-Ray Data for Other FU Ori Stars}

We compare X-ray constraints on Gaia 17bpi to properties of other FU Ori stars that were previously observed by XMMNewton or Chandra. We base this comparison on the list of FU Ori-type stars compiled by Connelley \& Reipurth (2018), which includes both bona fide outbursts as well as the FU Orilike sources that have spectroscopic features of FU Ori stars but did not have observed eruptions. ${ }^{2}$ Despite the passage of multiple decades since the outbursts of some of these objects, they are still elevated in brightness compared to their preoutburst level, albeit some have decayed significantly (e.g., V1057 Cyg; Clarke et al. 2005). Furthermore, all of the stars in the sample continue to have spectroscopic characteristics of FU Ori stars (Connelley \& Reipurth 2018).

Our sample of FU Ori stars includes all stars from the above list for which Chandra or XMM-Newton data is publicly available as of 2019 May. In addition to the published X-ray sources listed in Section 1, we also include unpublished archival X-ray data for V883 Ori, V2775 Ori, V900 Mon, V2494 Cyg, V2495 Cyg, V733 Cep, V1057 Cyg, V1515 Cyg, and IRAS 05450+0019, and V960 Mon (second epoch).

Depending on the availability of data for each FU Ori star, several methods have been used to assemble X-ray properties. For cases where model fits to the X-ray data have been published, we use these models to calculate the absorptioncorrected X-ray luminosities $L_{\mathrm{X}}$ in the $0.5-8.0 \mathrm{keV}$ band-i.e., the integrated X-ray luminosity of the model with absorption set to zero. In other cases, we make use of publicly available catalogs, such as the XMM Newton Serendipitous Source Catalog data release 7 (3XMM-DR7; Rosen et al. 2019) and the Star Formation in Nearby Clouds catalog (SFiNCs; Getman et al. 2017), as well as data from the XMM-Newton and Chandra archives, which we reduce using the standard recipes (Appendix A).

\section{Constraints on X-Ray Properties for Undetected Sources}

\subsection{Upper Limit on X-Ray Count Rate}

Most of the non-detections of FU Ori stars come from XMMNewton observations (13 out of 14). Eleven out of the 13 are located in regions of the sky covered by $3 X M M-D R 7$. For

\footnotetext{
2 Our final list includes 13 objects from the "bona fide" class as well as three FU Ori-like objects (L1551 IRS 5, IRAS 05450+0019, and Z CMa). We use both classes in this paper.
} 
Table 1

X-Ray Flux Upper Limits for Gaia 17bpi

\begin{tabular}{lrrrr}
\hline \hline \multicolumn{2}{c}{ Band } & & \multicolumn{3}{c}{ Flux } \\
\cline { 3 - 5 } $\begin{array}{l}E_{\min } \\
(\mathrm{keV})\end{array}$ & $\begin{array}{c}E_{\max } \\
(\mathrm{keV})\end{array}$ & $95 \%$ & $\begin{array}{c}99 \% \\
\left(\mathrm{erg} \mathrm{s}^{-1} \mathrm{~cm}^{-2}\right)\end{array}$ \\
\hline 0.2 & 0.5 & $2.7 \times 10^{-16}$ & $3.8 \times 10^{-16}$ & $5.3 \times 10^{-16}$ \\
0.5 & 1.0 & $3 \times 10^{-16}$ & $5 \times 10^{-16}$ & $7 \times 10^{-16}$ \\
1.0 & 2.0 & $6 \times 10^{-16}$ & $8 \times 10^{-16}$ & $1.1 \times 10^{-15}$ \\
2.0 & 4.5 & $1.7 \times 10^{-15}$ & $3 \times 10^{-15}$ & $4 \times 10^{-15}$ \\
4.5 & 12.0 & $8 \times 10^{-15}$ & $1.1 \times 10^{-14}$ & $1.6 \times 10^{-14}$ \\
\hline
\end{tabular}

these objects, the FLIX tool $^{3}$ (Carrera et al. 2007, their Appendix A) is used to calculate upper limits on flux in the 5 XMM-Newton energy bands (Table 1) at each stars' position based on the X-ray images that were used to generate the 3XMM-DR7 catalog.

The other two XMM-Newton non-detections include our observation of Gaia 17bpi and a recent observation of V900 Mon, which we analyze using the same method as detailed below for Gaia 17bpi. The final non-detection is a Chandra observation of V733 Cep, for which we use the completeness limit of the SFiNCs catalog. Details of the process for individual FU Ori stars are further described in Appendix A.2.

We compute upper limits on the flux of Gaia 17bpi in each of the bands listed above using the data from the pn detector. In each band, the upper limits for the number of X-ray events from Gaia $17 \mathrm{bpi}\left(N_{\star}\right)$ are determined by counting the number of events in the source extraction region $\left(N_{\text {ext }}\right)$ and comparing this to numbers of events in background regions $\left(N_{\mathrm{bgd}}\right)$. We assume that the number of events in each extraction region is drawn from a Poisson distribution, and we find that the variations in the numbers of counts in each of the nine background regions are consistent with this assumption. Then, the likelihood of $N_{\star}$ given that $N_{\text {ext }}$ counts were observed would be the Poisson probability

$$
p\left(N_{\text {ext }} \mid N_{\star}\right)=\frac{\left(N_{\star}+N_{\text {bgd }}\right)^{N_{\text {ext }}} e^{-\left(N_{\star}+N_{\text {bgd }}\right)}}{N_{\text {ext }} !} .
$$

As few FU Ori-type stars have been detected in the X-ray, the existing fluxes provide little guidance for what to use for a prior. For pre-main-sequence stars, studies of the Orion Nebula Cluster reveal a distribution that is relatively constant in $\log \mathrm{X}$-ray luminosity $\left(\log L_{\mathrm{X}}\right)$ over the range from $10^{28}$ to $2 \times 10^{30} \mathrm{erg} \mathrm{s}^{-1}$, and decreases for higher luminosities (Feigelson et al. 2005). For our prior distribution on the flux of Gaia 17bpi, we used a uniform distribution in $\log F_{\mathrm{X}}$ between -17.0 and -12.5 , which spans the full range of plausible upper limits. However, we found that if we use an alternative prior, generated by adaptively smoothing the distribution of X-ray fluxes of stars in the Orion sample, the results are very similar.

From Bayes' theorem we know,

$$
p\left(N_{\star}\right)=\frac{p\left(N_{\mathrm{ext}} \mid N_{\star}\right) p\left(N_{\star}\right)}{\int_{0}^{\infty} p\left(N_{\mathrm{ext}} \mid N_{\star}\right) p\left(N_{\star}\right) d N_{\star}} .
$$

The integral in the denominator can be computed numerically, allowing us to compute the credible interval on the range $\left[0, N_{\star, \mathrm{ul}}\right]$ that contains $99 \%$ of the probability, where $N_{\star, \mathrm{ul}}$ is the

\footnotetext{
3 https://www.ledas.ac.uk/flix/flix_dr7.html
}

99\% upper limit. We also compute $95 \%$ and $99.9 \%$ upper limits.

The upper limits on $N_{\star}$ are converted to count rate by dividing by ontime $(32,694 \mathrm{~s}$ at the position of Gaia 17bpi). Count rate is converted to flux by dividing by the mean value of the ARF in the band and multiplying by the central energy (see approximation by Broos et al. 2010, their Equation (8)). The $95 \%, 99 \%$, and $99.9 \%$ upper limits on flux are reported in Table 1. Flux limits are less restrictive for the higher energy bands because the pn detector is less sensitive to photons in these energy ranges and the flux would be distributed among fewer photons.

\subsection{Constraints on $L_{\mathrm{X}}, k T$, and $N_{\mathrm{H}}$}

The upper limits on X-ray fluxes can be used to determine which combinations of X-ray luminosity, plasma temperature, and absorbing column are possible. For the non-detections, we calculate upper limits on X-ray luminosity as a function of $N_{\mathrm{H}}$ and $k T$. The procedure is described in detail below for Gaia 17bpi, and this case is illustrated in Figure 1. The limits for other sources are summarized in Table 2.

We use the Portable, Interactive Multi-Mission Simulator (PIMMS; Mukai 1993) to calculate X-ray flux in the five $X M M$-Newton bands for a grid of temperatures $(k T=0.25,0.8$, $1.5,3.0,6.0$, and $10.0 \mathrm{keV})$, absorptions $\left(N_{\mathrm{H}}=10^{21}\right.$ to $10^{24} \mathrm{~cm}^{-2}$ ), and intrinsic (i.e., unabsorbed) X-ray luminosities $\left(L_{\mathrm{X}}=10^{28}\right.$ to $10^{33} \mathrm{erg} \mathrm{s}^{-1}$ in the $0.5-8.0 \mathrm{keV}$ band), given the distances to the FU Ori stars from Appendix B. For these simulations, we set metal abundances of the model to 0.4 times solar, which are typical for X-ray emission from YSOs (e.g., Imanishi et al. 2001; Feigelson et al. 2002); however, this assumption has little effect on the resulting limits. A combination of $L_{\mathrm{X}}, k T$, and $N_{\mathrm{H}}$ is ruled out if it produces a flux in one of the five bands that exceeds the flux limits from Table 1.

The colored lines in Figure 1 show limits on $L_{X}$ for Gaia $17 \mathrm{bpi}$ as a function of $N_{\mathrm{H}}$. Each color shows limits for a different temperature plasma, while the dotted, solid, and dashed lines refer to the $95 \%, 99 \%$, and $99.9 \%$ limits, respectively. Other symbols and annotations on the graph are explained in Sections 3.3-4 below.

First, we examine whether a lower-temperature plasma ( $k T \sim 0.25 \mathrm{keV}$, as would be expected for accretion shocks or jets) would have been detectable. If the source were lightly absorbed $\left(N_{\mathrm{H}}<10^{21.6} \mathrm{~cm}^{-2}\right)$, it must be less luminous than $\sim 10^{29}-10^{30} \mathrm{erg} \mathrm{s}^{-1}$. However, for somewhat higher absorption, there is little constraint on the possible luminosity of a soft $\mathrm{X}$-ray source.

For higher-temperature plasmas $(k T \sim 0.8-3.0 \mathrm{keV}$, typical for coronal X-ray emission from $\mathrm{T}$ Tauri stars), X-ray luminosities above $\sim 10^{30} \mathrm{erg} \mathrm{s}^{-1}$ can be excluded if the absorption is not much greater than $N_{\mathrm{H}}=10^{22} \mathrm{~cm}^{-2}$, as inferred from the optical pre-outburst observations (Section 3.3). However, if the postoutburst absorption were greater, the range of possible X-ray luminosities increases with increasing $N_{\mathrm{H}}$.

For very hot plasmas $(k T>10 \mathrm{keV}$, similar the the hottest $\mathrm{X}$-ray emission from $\mathrm{T}$ Tauri stars or X-ray emission from some FU Ori stars), possible X-ray luminosities range from $<10^{30} \mathrm{erg} \mathrm{s}^{-1}$ for light absorption to $<10^{30.8} \mathrm{erg} \mathrm{s}^{-1}$ for the heavy extinction of $N_{\mathrm{H}} \sim 10^{23.5} \mathrm{~cm}^{-2}$ (equivalent to $A_{V} \sim 140$ mag with a typical gas-to-dust law). 


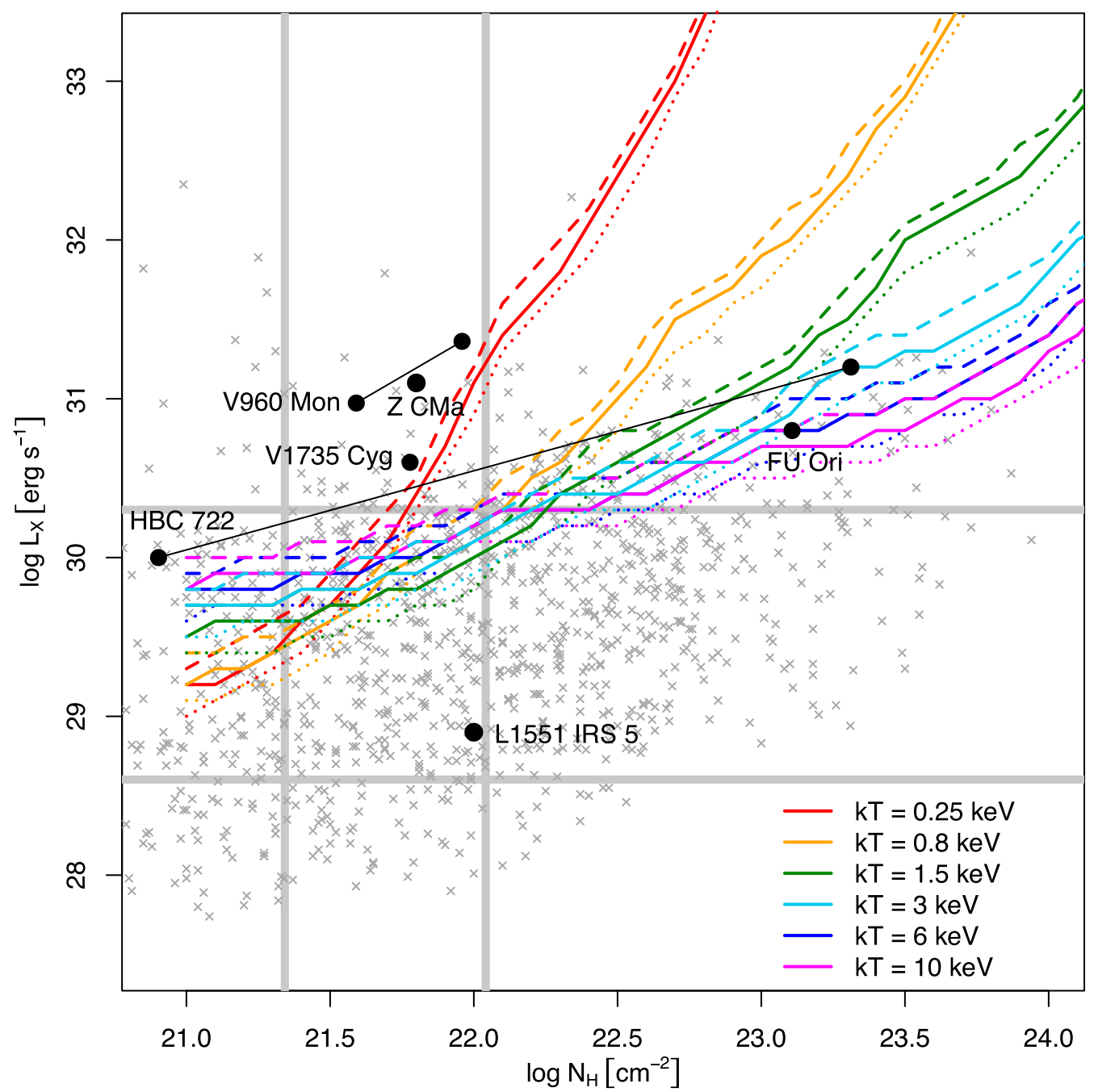

Figure 1. The colored lines show the maximum possible $L_{\mathrm{X}}$ for Gaia $17 \mathrm{bpi}$ as a function of $N_{\mathrm{H}}$ for various assumptions of plasma temperature-red: $k T=0.25 \mathrm{keV}$, orange: $0.8 \mathrm{keV}$, green: $1.5 \mathrm{keV}$, cyan: $3 \mathrm{keV}$, blue: $6 \mathrm{keV}$, and magenta: $10 \mathrm{keV}$. The dotted, solid, and dashed lines indicated the $95 \%$, $99 \%$, and $99.9 \%$ probability limits, respectively. The light gray vertical lines indicate the range of $N_{\mathrm{H}}$ that would correspond to the pre-burst $A_{V}=1-5$ mag estimated by Hillenbrand et al. (2018), while the horizontal lines show the probable range of pre-burst $L_{\mathrm{X}}$ estimated from the $L_{\mathrm{X}}-L_{\mathrm{bol}}$ relation. The gray X's show $L_{\mathrm{X}}$ and $N_{\mathrm{H}}$ for low-mass, non-flaring premain-sequence stars in the Orion Nebula Cluster (Getman et al. 2005), while the black points indicated previously detected FU Ori-type stars. The black lines connect points for FU Ori stars observed at different epochs.

The curves of $L_{\mathrm{X}}$ upper limits for the other FU Ori stars with non-detections look similar to those of Gaia 17bpi, with vertical shifts resulting from different distances and integration times. In order to compare upper limits for different objects, it is helpful to pick a fiducial $\left(N_{\mathrm{H}}, k T\right)$ combination. We choose $k T=3 \mathrm{keV}$ and $N_{\mathrm{H}}=10^{21.8} \mathrm{~cm}^{-2}$, and we tabulate the upper limits on $L_{\mathrm{X}}$ in Table 2 using these assumptions. These temperatures and absorptions are close to the median values obtained from the sample of six FU Ori stars where these quantities have been measured (also shown in Table 2). However, it is possible that the upper limits on $L_{\mathrm{X}}$ in Table 2 would be too low if $N_{\mathrm{H}}$ were much greater than $N_{\mathrm{H}}=10^{21.8} \mathrm{~cm}^{-2}$-implications of the $\left(N_{\mathrm{H}}, k T\right)$ choice are discussed in Section 5.

\subsection{Expected Ranges of Pre-outburst $\mathrm{N}_{H}$ and $\mathrm{L}_{X}$ for Gaia $17 b p i$}

Gaia 17bpi was not observed in the X-ray prior to the outburst. However, probable ranges for pre-outburst $N_{\mathrm{H}}$ and $L_{\mathrm{X}}$ can be estimated from statistical relations between X-ray properties and stellar properties for $\mathrm{T}$ Tauri stars. The preoutburst spectral energy distribution (SED) of Gaia 17bpi could be fit by a variety of reddened stellar photospheric models with $A_{V}$ ranging from 1 to $5 \mathrm{mag}$ and corresponding $T_{\text {eff }}$ ranging from 2900 to $4500 \mathrm{~K}$ (Hillenbrand et al. 2018), with a preferred model with $T_{\text {eff }}=3500 \mathrm{~K}$ and $A_{V}=3 \mathrm{mag}$ (see also Appendix C). From these ranges, we derive extinction-corrected bolometric luminosities of $L_{\mathrm{bol}} \approx 4 \times 10^{32}-2 \times 10^{33} \mathrm{erg} \mathrm{s}^{-1}$ for the preoutburst star using bolometric and color corrections from Pecaut \& Mamajek (2013).

Ryter (1996) found $N_{\mathrm{H}} / A_{V} \approx 2.2 \times 10^{21} \mathrm{~cm}^{-2} / \mathrm{mag}$ for Galactic extinction, and this law provides a reasonable description for the observed relation between $A_{V}$ and $N_{\mathrm{H}}$ for T Tauri stars (e.g., Feigelson et al. 2005; Kuhn et al. 2010). For Gaia 17bpi, this would yield a pre-burst $N_{\mathrm{H}}=2.2 \times 10^{21}-1.1 \times 10^{22} \mathrm{~cm}^{-2}$. This range is indicated in Figure 1 for comparison.

$\mathrm{X}$-ray luminosity is correlated with bolometric luminosity (or mass) for T Tauri stars, albeit with significant scatter. From the 
Table 2

Summary of X-Ray Properties of FU Ori Stars

\begin{tabular}{|c|c|c|c|c|c|c|c|c|}
\hline Name & $\begin{array}{l}\log L_{\mathrm{X}}^{\mathrm{a}} \\
\left(\mathrm{erg} \mathrm{s}^{-1}\right)\end{array}$ & $\begin{array}{c}k T^{b} \\
(\mathrm{keV})\end{array}$ & $\begin{array}{l}\log N_{\mathrm{H}} \\
\left(\mathrm{cm}^{-2}\right)\end{array}$ & Facility & $\begin{array}{l}\text { Date }^{c} \\
\text { (UT) }\end{array}$ & X-ray Ref. ${ }^{\mathrm{d}}$ & Region & $\begin{array}{l}\text { Dist. }^{e} \\
(\mathrm{pc})\end{array}$ \\
\hline Gaia 17bpi & $<29.9$ & {$[3.0]$} & {$[21.8]$} & XMM & 2018 Nov 15 & this work & G53.2 & 1200 \\
\hline $\mathrm{Z} \mathrm{CMa}$ & 31.1 & $0.4+7.5$ & 21.8 & $\mathrm{CXO}$ & $2003 \operatorname{Dec} 7$ & Stelzer et al. (2009) & CMa R1 & 1120 \\
\hline V960 Monocerotis & 31.0 & 2.6 & 21.6 & $\mathrm{CXO}$ & 2015 Jan 26 & this work & CMa R1 & 1120 \\
\hline$\cdots$ & 31.4 & $0.5+2.6$ & 22.0 & XMM & 2016 Oct 28 & this work & $\cdots$ & $\cdots$ \\
\hline V1515 Cygni & $<29.9$ & {$[3.0]$} & {$[21.8]$} & XMM & 2006 Oct 22 & 3XMM-DR7 & NGC 6914 & 960 \\
\hline V1057 Cygni & $<29.9$ & {$[3.0]$} & {$[21.8]$} & XMM & 2005 Nov 26 & 3XMM-DR7 & NGC 7000 & 795 \\
\hline HBC 722 & $<30.8$ & {$[3.0]$} & {$[21.8]$} & XMM & 2010 Nov 25 & 3XMM-DR7 & NGC 7000 & 795 \\
\hline$\cdots$ & 30.0 & 8.4 & 20.9 & XMM & 2011 May 26 & Liebhart et al. (2014) & $\cdots$ & $\cdots$ \\
\hline$\cdots$ & 31.2 & 2.3 & 23.3 & $\mathrm{CXO}$ & 2013 Jul 17 & Liebhart et al. (2014) & $\cdots$ & .. \\
\hline V1735 Cygni & 30.6 & 13.7 & 21.8 & XMM & 2006 Jul 22 & Skinner et al. (2009) & IC 5146 & 752 \\
\hline V2494 Cygni & $<29.6$ & {$[3.0]$} & {$[21.8]$} & XMM & $2013 \operatorname{Dec} 6$ & 3XMM-DR7 & Cyg OB7 & 594 \\
\hline FU Orionis & 30.8 & 5.58 & 23.1 & XMM & 2004 Mar 8 & Skinner et al. (2006) & $\lambda$ Ori & 404 \\
\hline$\cdots$ & 30.8 & 4.50 & 23.1 & $\mathrm{CXO}$ & 2008 Nov 24 & Skinner et al. (2010) & $\cdots$ & $\cdots$ \\
\hline V883 Orionis & $<29.7$ & {$[3.0]$} & {$[21.8]$} & XMM & 2004 Mar 27 & 3XMM-DR7 & Orion A & 392 \\
\hline$\cdots$ & $<29.6$ & {$[3.0]$} & {$[21.8]$} & XMM & 2004 Aug 25 & 3XMM-DR7 & $\cdots$ & $\cdots$ \\
\hline L1551 IRS 5 & 28.9 & 0.6 & 22.0 & $\mathrm{XMM}+\mathrm{CXO}$ & 2000 Sep 9 & Schneider et al. (2011) & L1551 & 144 \\
\hline
\end{tabular}

Notes. Stars are sorted in inverse order of distance.

a Absorption-corrected X-ray luminosities. All values are adjusted for assumed distance and to a common X-ray band (0.5-8.0 keV).

${ }^{\mathrm{b}}$ Brackets around values indicate that they are assumed, not derived from X-ray spectra.

c Start time of the first observation used for the analysis.

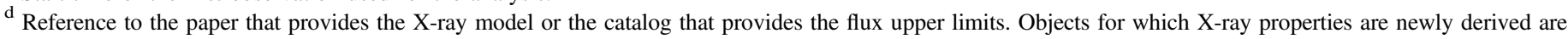
labeled "this work."

e See Appendix B for justification of adopted distances, which includes original work.

$L_{\mathrm{X}}-L_{\mathrm{bol}}$ relation ${ }^{4}$ in Gregory et al. (2016), we would expect a pre-outburst X-ray luminosity to fall in the range $L_{\mathrm{X}}=4 \times$ $10^{28}-2 \times 10^{30} \mathrm{erg} \mathrm{s}^{-1}$ taking into account both uncertainty on the model and statistical scatter. Given that this range spans the upper limit on $L_{\mathrm{X}}$ of Gaia 17bpi as indicated on Figure 1, our constraints do not reveal whether X-ray flux was suppressed by the beginning of the outburst relative to the expected preoutburst emission.

\subsection{Comparison with Generic YSOs}

We use a sample of pre-main-sequence stars detected in the Chandra Orion Ultradeep Project (COUP; Feigelson et al. 2005; Getman et al. 2005) as a representative sample of generic YSOs. We include only cluster members that are low-mass (excluding stars with spectral-types of B and earlier), that do not have major X-ray flares during the observation (the list of flaring sources is taken from Getman et al. 2008), and have measurements of $L_{\mathrm{X}}$ and $N_{\mathrm{H}}$. This yields a sample of 1200 objects that we refer to as the "COUP sample."

The COUP sample is plotted on Figure 1. These stars (gray x's) mostly lie within the same region of the plot as the permitted Gaia 17bpi X-ray luminosities; however, some of the most luminous COUP sample $\mathrm{X}$-ray sources would have been detected in this observation. This suggests that if Gaia 17bpi

\footnotetext{
4 We use the regression line and standard deviation derived for "all stars" in Table 4 of Gregory et al. (2016).
}

did have X-ray properties that were unchanged by the outburst, it would most likely not have been detected.

\section{X-Ray Properties of the Full FU Ori Sample}

In our full sample, six FU Ori stars were detected in the X-ray, while ten were not (Table 2). Several stars were observed at multiple epochs during the outburst or shortly afterwards, and these observations are listed separately in the table in order to document any evolution of X-ray properties during an outburst (see also Appendix A). The detected FU Ori stars have quiescent $\mathrm{X}$-ray light curves, with the possible exception of the 2008 Chandra epoch of FU Ori, where X-ray variability is interpreted by Skinner et al. (2010) as a possible flare.

The six detected FU Ori-type stars are plotted in Figure 1 (black points). Five of these lie above the $L_{\mathrm{X}}$ curve for Gaia 17bpi for the corresponding $k T$ on this figure, indicating that they would have been detected if they were in this observation at the same distance as Gaia 17bpi. This implies that Gaia 17bpi is currently fainter in the X-ray than these five stars. These five stars are also much brighter than the typical YSOs in the COUP sample in the Orion Nebula Cluster, meaning that they either represent the high-luminosity tail of the pre-main-sequence star XLF, or their X-ray luminosities have been enhanced by their outbursts. One object, L1551 IRS 5, has an $L_{\mathrm{X}}$ much lower than the other detected FU Ori stars. 


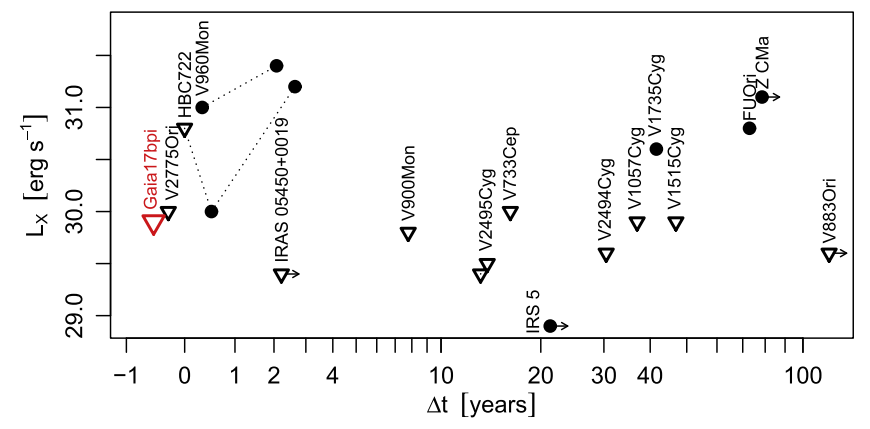

Figure 2. X-ray luminosities of FU Ori-type stars observed with Chandra or $X M M-N e w t o n$ plotted as a function to the time $\Delta t$ between the peak of the optical outburst and X-ray observation. Detections are shown as filled circles and non-detections as downward-pointing triangles. Arrows indicate that the time since outburst is a lower limit for four objects. Objects that were observed at multiple epochs are joined with dashed lines. The $\Delta t$ axis is approximately linear for $\Delta t \approx 0$ and becomes logarithmic for larger $\Delta t$ due to the large dynamic range in time separation.

\subsection{Time Evolution of X-Ray Luminosity}

Figure 2 shows X-ray luminosities (or upper limits on X-ray luminosity) for the FU Ori stars as a function of the time elapsed between the peak of the optical outburst and the X-ray observation. The dates of the outburst peaks are taken from Connelley \& Reipurth (2018); however, in many cases, these are not well constrained due to either the cadence of observing, ambiguity in the shape of the light curve, or (in four cases) no observation from before the outburst. In the case of Gaia 17bpi (shown in red), we use the date of the brightest $G$-band magnitude, 2019 May 29. However, at the time of writing, there is no indication that the light curve has reached its peak.

No obvious trend in X-ray luminosity as a function of time since outburst is observed in the sample. Highly X-ray luminous FU Ori stars were observed both around the time of the optical peak ( $\Delta t<3 \mathrm{yr}$ ) as well as decades later (40< $<t<100 \mathrm{yr}$ ). There are also non-detections of FU Ori stars spanning nearly the whole range of the post-peak phase. The two observations made before the outburst peak (Gaia $17 \mathrm{bpi}$ and V2775 Ori) both resulted in non-detections, with fairly low $L_{\mathrm{X}}$ limits $\left(<10^{30} \mathrm{erg} \mathrm{s}^{-1}\right)$ given our assumptions on $N_{\mathrm{H}}$ and $k T$. An observation of HBC 722 at the approximate time of the peak also yielded a non-detection, albeit with a higher upper limit.

Both of the FU Ori stars that were observed multiple times shortly after the outburst peak (HBC 722 and V960 Mon) increased in luminosity with the subsequent observations. Furthermore, the detection of high- $L_{\mathrm{X}}$ FU Ori stars at late times suggest that an enhancement of X-ray luminosity can be long lasting. However, more data are needed before a definitive conclusion can be made about the time evolution of FU Ori stars in the various stages of their life cycles.

\subsection{XLF of FU Ori Stars}

One method of examining the effect of an FU Ori outburst on X-ray emission from a YSO is through comparison of the XLF for FU Ori stars to ordinary YSOs. For this comparison, we use the COUP sample, which has a well-studied XLF (Feigelson et al. 2005) that provides a good match to the shape of the distribution of X-ray luminosities of YSOs in other starforming regions (Wang et al. 2008; Kuhn et al. 2015).

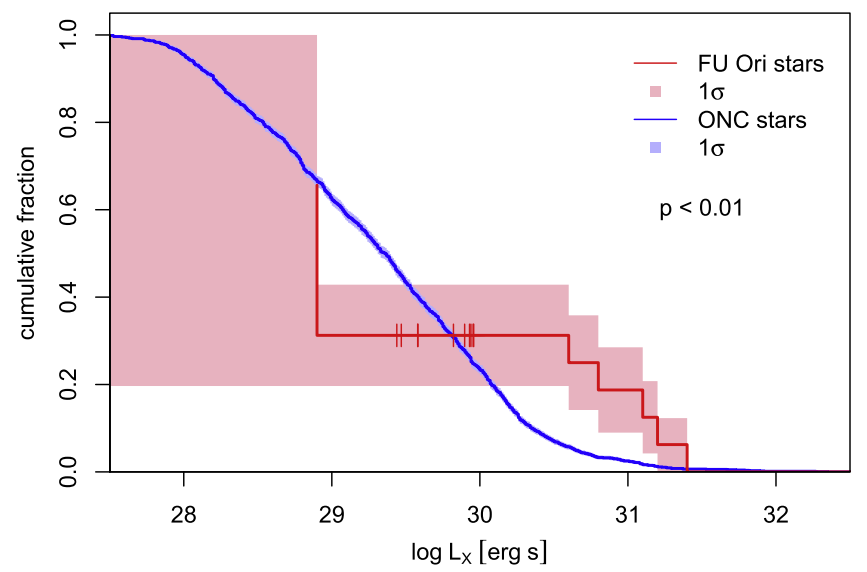

Figure 3. Cumulative distributions of $L_{X}$ for FU Ori-type stars (red) and the COUP samples (blue) estimated using the Kaplan-Meier statistic. Tic marks indicate upper limits, and steps indicate detections for FU Ori stars. Upper limits derived for COUP stars are included in the derivation of the line, but the tic marks are suppressed on the plot to avoid clutter. The envelopes around both lines indicate uncertainty.

The Kaplan-Meier statistic (Kaplan \& Meier 1958) can be used to estimate the cumulative distribution function for a data set with censoring, in this case, censoring of low values of X-ray luminosity as a result of limits on detector sensitivity (see Feigelson \& Nelson 1985). Figure 3 shows the KaplanMeier estimator for X-ray luminosities-steps are drawn at the value of detected sources, and tic marks indicate upper limits on $L_{\mathrm{X}}$, and the shaded region shows the standard deviation of the statistic. For objects that have multiple entries in Table 2, we base the following analysis on the most recent good measurement of $L_{X} \cdot{ }^{5}$ Due to the small number of sources, the uncertainty in the shape of the distribution is large and becomes unconstrained for X-ray luminosities below the least luminous detected source. The Kaplan-Meier estimator is also shown for the sample of COUP X-ray sources (Section 3.4), with upper limits determined for undetected stars (not shown on the plot) from Getman et al. (2005, their Table 11). Due to the large size of the COUP sample, the standard deviation of the KaplanMeier statistic for these data is nearly invisible on the scale of the plot.

The estimated FU Ori XLF shows that about one-third of the FU Ori stars have X-ray luminosities $>10^{30.5} \mathrm{erg} \mathrm{s}^{-1}$. However, there is a considerable gap, by about an order of magnitude in $L_{\mathrm{X}}$, between the high X-ray luminosity sources and many of the upper limits. The location of the upper limits suggests that the bottom two-thirds of the FU Ori population in $L_{\mathrm{X}}$ have luminosities consistent with those of typical YSOs.

Log-rank tests are typically used to test for differences in distributions with censored values, with different weighting schemes giving rise to different types of tests. We are particularly interested in differences in distributions at high luminosities, so that we can determine whether FU Ori stars tend to be more X-ray luminous than T Tauri stars. However, many of the tests, such as the log-rank test with no weighting, would be more sensitive to the low-luminosity end of the distribution. We chose the $G-\rho$ class of tests by Harrington \& Fleming (1982), with $\rho=10$, implemented by the function survdiff in the $R$ package survival (Therneau \& Grambsch 2000). We note that this choice

\footnotetext{
5 This excludes the 2008 epoch for FU Ori showing a possible flare and the non-constraining upper limit from the 2014 epoch for V733 Cep. However, these exclusions do not have a significant effect on the results.
} 
of $\rho$ is somewhat unusual because it puts significant emphasis on the high-luminosity end of the distribution; nevertheless it is still a valid test statistic. ${ }^{6}$

Given the small number of FU Ori stars, we calculate the $p$ value for the hypothesis test using simulations of the test statistic, rather than assuming the asymptotic distribution calculated by Harrington \& Fleming (1982). To simulate observations of FU Ori stars (assuming the null hypothesis that the distribution of X-ray luminosities for the FU Ori sample and the COUP sample are drawn from the same underlying distribution), we randomly draw $16 L_{\mathrm{X}}$ values from the COUP sample and censor them based on the detection limits determined for each observation of the actual FU Ori stars, from which we compute the test statistic. From 10,000 simulations, we are able to reject the null hypothesis with $p<0.01$.

This result can be seen more intuitively using the following, alternative probabilistic argument. From the estimated cumulative distribution of COUP $L_{\mathrm{X}}$ values, it can be seen that only $7 \%$ of objects in the COUP sample have $L_{X}>10^{30.5} \mathrm{erg} \mathrm{s}^{-1}$, while five out of $16 \mathrm{FU}$ Ori stars do. If the $L_{\mathrm{X}}$ values for the FU Ori stars are randomly drawn from the same distribution as the COUP stars, the probability of drawing five or more stars with $L_{\mathrm{X}}>10^{30.5} \mathrm{erg} \mathrm{s}^{-1}$ would be

$$
p=\sum_{k=5}^{16} 0.07^{k} 0.93^{(16-k)}\left(\begin{array}{c}
16 \\
k
\end{array}\right) \approx 0.004 .
$$

The results of both tests indicate that the XLF for FU Ori stars differs from that of $\mathrm{T}$ Tauri stars, in the sense of FU Ori stars being more luminous. The result using the Harrington-Fleming test remains statistically significant even if only four stars were considered to have $L_{\mathrm{X}}>10^{30.5}$ (for example, if we used $L_{\mathrm{X}}$ values from the first $\mathrm{X}$-ray observations rather than the most recent, turning HBC 722 into a non-detection).

For the COUP sample, the top $30 \%$ most luminous stars have $L_{\mathrm{X}}>10^{29.8} \mathrm{erg} \mathrm{s}^{-1}$ based on the Kaplan-Meier estimator for the distribution. Given that the top 30\% FU Ori stars have $L_{\mathrm{X}}>10^{30.5} \mathrm{erg} \mathrm{s}^{-1}$, this suggests that the enhancement of $\mathrm{X}$-ray luminosity for the X-ray luminous FU Ori stars is $\sim 0.7$ dex (or roughly a factor of five).

\subsection{Effects of Mass and YSO Class on $\mathrm{L}_{X}$}

Masses of stars undergoing FU Ori outbursts are poorly constrained due, in most cases, to a lack of pre-outburst spectroscopy. Modeling of post-outburst SEDs, which are dominated by disk emission, provides indirect estimates of stellar properties, suggesting that most of these stars are lowmass (Hartmann \& Kenyon 1996; Gramajo et al. 2014).

Figure 4 shows the XLF for FU Ori stars (full sample) compared to stars in the COUP sample that have been stratified by mass. For COUP stars, we use masses from Da Rio et al. (2012) derived using the Baraffe et al. (1998) evolutionary models and divide stars into groups with mass ranges $M \leqslant 0.25 M_{\odot}, 0.25<M \leqslant 0.5 M_{\odot}, 0.5<M \leqslant 1.0 M_{\odot}$, and

\footnotetext{
6 We are most interested in the high-luminosity tail of the distribution, i.e., whether there are significantly more FU Ori stars that have X-ray luminosities above the $\sim 95 \%$ quantile of T Tauri star luminosities. For the HarringtonFleming test, weights are defined as $w_{i}(\rho)=\left[\hat{S}\left(L_{\mathrm{X}, i}\right)\right]^{\rho}$, where $\hat{S}$ is the pooled survival probability at the luminosity $L_{\mathrm{X}, i}$ of the $i$ th star. For this end of the distribution, typical values of weights would be $w \sim 0.95^{10} \sim 0.6$, which is reasonable. The test with this choice of $\rho$ would be relatively insensitive to differences in the distributions below the $70 \%$ quantile of $L_{\mathrm{X}}$.
}

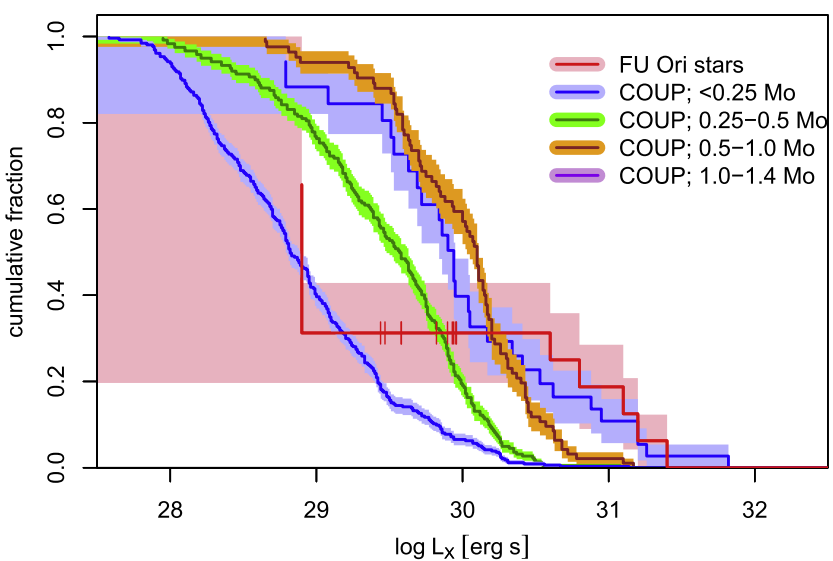

Figure 4. The red line showing the Kaplan-Meier estimator for the FU Ori $\mathrm{X}$-ray luminosity function is that same as in Figure 3, but this is compared to COUP stars in restricted mass ranges.

$1.0<M \leqslant 1.4 M_{\odot}$. The different mass strata have different luminosity functions, reflecting the well-known $L_{\mathrm{X}}$-mass dependence (Preibisch et al. 2005). The two lowest-mass strata in the COUP sample contain very few stars with $L_{\mathrm{X}}>$ $10^{30.5} \mathrm{erg} \mathrm{s}^{-1}$ so the difference between them and the FU Ori stars is statistically significant $(p<0.001)$. Even the $0.5<$ $M \leqslant 1.0 M_{\odot}$ COUP sample has significantly fewer high X-ray luminosity sources than the FU Ori sample $(p=0.006)$. The FU Ori stars most closely follow the distribution for stars with $1.0<M \leqslant 1.4 M_{\odot}(p=0.8)$. However, it seems unlikely that all five X-ray luminous FU Ori stars have masses this high, given that most of them have mass estimates lower than $1 M_{\odot}$ (Gramajo et al. 2014).

FU Ori stars have been classified by their SED shapes as either Class I or Class II YSOs, with a predominance of Class I objects (Gramajo et al. 2014). This raises the question of how SED stage affects X-ray luminosity and whether this could be the cause of higher $L_{\mathrm{X}}$ seen for FU Ori stars. Out of the five FU Ori stars with $L_{\mathrm{X}}>10^{30.5} \mathrm{erg} \mathrm{s}^{-1}$, FU Ori, Z CMa, and HBC 722 are likely Class I, and V1735 Cyg and V960 Mon are likely Class II (Gramajo et al. 2014; Kóspál et al. 2015). The mixture in classes suggests that class alone does not account for the higher $L_{X}$. Furthermore, it is thought that $\mathrm{X}$-ray emission is suppressed in both Class I and Class II YSOs relative to Class III YSOs (Telleschi et al. 2007; Prisinzano et al. 2008), making it less likely that the enhancement of $L_{\mathrm{X}}$ for FU Ori stars can be explained by YSO class alone.

One could ask whether the YSOs in the Orion Nebula Cluster are representative of X-ray properties of all young stars in all starforming environments, and, if there is a difference, how it would affect the comparison between our sample of FU Ori stars and generic YSOs. Kuhn et al. (2015) examined the XLF of YSOs in 17 nearby massive star-forming regions, including the COUP sample as well as samples from regions that contain younger stars, like W40 and NGC 6334, and older stars, like NGC 2362. Overall, the XLFs for the different regions appear to have similar shapes over the range of $L_{\mathrm{X}}=10^{30.5}-10^{31.5} \mathrm{erg} \mathrm{s}^{-1}$ (their Figure 1), with the exception that some regions with stars older than $\sim 5 \mathrm{Myr}$ have steeper drop-offs with luminosity. None of the regions (including those with large numbers of Class I objects) appear to have a relative excess of high X-ray luminosity stars compared to Orion. Thus, it is unlikely that the T Tauri stars in any of these regions would more closely resemble the FU Ori XLF. 


\subsection{The Effect of X-Ray Flares on $\mathrm{L}_{X}$ Distributions}

The X-ray light curves for the FU Ori stars do not show evidence of large-amplitude flares, with the possible exception of the 2008 epoch for FU Ori (Skinner et al. 2010). Thus, in the previous sections, we compared the FU Ori sample to the sample of low-mass stars in COUP without significant flare activity. The flaring stars in COUP are among the most X-ray luminous sources in the sample, so inclusion of these sources would broaden that tail of the COUP luminosity function, possibly making the difference between the FU Ori XLF and COUP XLF less significant. To investigate this, we repeat the Harrington-Fleming test from above, this time including both non-flaring and flaring COUP sources. We find $p=0.02$, which is still marginally significant, but not as strong an effect as when non-flaring sources are compared.

\section{Discussion and Conclusion}

We used a sample of FU Ori stars observed by XMM-Newton and Chandra to investigate whether there is statistical evidence that the outbursts of these stars affect their X-ray emission. The data include a new $\sim 35 \mathrm{ks} X M M-N e w t o n$ observation of the most recent FU Ori outburst, Gaia 17bpi, as well as previously published and archival observations of 15 other FU Ori-type outbursts, yielding a sample (including upper limits for nondetections) that include over half of all known FU Ori stars.

Our analysis shows that the most luminous X-ray sources in our FU Ori sample - the five sources with $L_{\mathrm{X}}>10^{30.5} \mathrm{erg} \mathrm{s}^{-1}$ out of 16 objects-are too luminous to have been drawn by chance from the distribution of $L_{\mathrm{X}}$ for generic YSOs. These stars comprise about $30 \%$ of the FU Ori sample, making them a factor of $\sim 5$ times more X-ray luminous than the top $30 \%$ of generic YSOs. This effect seems counterintuitive because other, less-extreme cases of heightened accretion generally have the opposite effect of diminishing X-ray emission (Telleschi et al. 2007; Prisinzano et al. 2008). However, careful analysis of the full data set shows that high $L_{X}$ values noted by earlier studies of individual objects (e.g., Skinner et al. 2009) are common for FU Ori stars.

Although the data show a statistically significant effect, they do not yet indicate a cause-and-effect relation. One possibility is that the outbursts are the cause of the high X-ray emission; another is that high X-ray emission could increase the chance of an outburst; and finally, both X-ray emission and FU Ori outburst could be connected to a common underlying YSO property.

The high temperatures found from X-ray spectral fitting can be used to rule out one of the simplest explanations for the heightened X-ray emission-accretion shocks. Gas accreting onto a young star at approximately the freefall velocity can shock heat plasma to at most several million Kelvin (Hartmann et al. 2016, their Equation (4)), which is much less than the temperatures measured for the five most luminous FU Ori stars in Table 2, which range from $\sim 2 \times 10^{7}$ to more than $10^{8} \mathrm{~K}$. Instead, such temperatures are similar to those measured for magnetically heated coronae of pre-main-sequence stars. Nevertheless, even moderate $N_{\mathrm{H}}$ can obscure soft X-ray emission (e.g., Figure 1), so whether a soft component also exists is left relatively unconstrained.

If the outburst of an FU Ori stars is the cause of heightened $\mathrm{X}$-ray emission, it would mean that the eruption leads to a reconfiguration of the magnetosphere in a way that enhances
$\mathrm{X}$-ray emission. This may come about if the disturbance of a star's magnetosphere by the inward advance of the disk enhances reconnection. Furthermore, X-rays could be generated by strengthened magnetic fields in the disk (Liebhart et al. 2014). However, there has been little theoretical guidance on how FU Ori outbursts affect X-ray production.

On the other hand, it is also plausible that FU Ori outbursts could either be triggered directly by the high X-ray emission of a young star or by the strong magnetic activity that gives rise to the X-ray emission. Theoretical models of accretion outbursts suggest that magnetic fields and the ionization structure of the disk play key roles in these outbursts (Zhu et al. 2010; Bae et al. 2013, 2014), although these models have not investigated how changes in external sources of ionization would affect outbursts. However, a study of X-ray flares, which are comparable in X-ray luminosity to the most luminous FU Ori stars, suggests that they can have a considerable effect on the structure of the dead zone in disks (Ilgner \& Nelson 2006). In light of this, it is worth investigating whether FU Ori outbursts occur at a higher rate in stars that naturally have higher X-ray luminosities.

Although the enhancement of $L_{\mathrm{X}}$ seen for five of the FU Ori stars is statistically significant, it does not appear to be universal, and there is a fairly large gap of $>0.5$ dex between these five stars and the remaining 11 stars-assuming that these 11 have moderate absorbing column densities. However, the non-detections of some stars cannot be considered evidence for suppression of X-ray emission because we would not expect to detect most ordinary YSOs at the distances of the FU Ori stars given the integration times that were used (e.g., Section 3.3). Furthermore, even an FU Ori star with $L_{\mathrm{X}} \sim 10^{30.5} \mathrm{erg} \mathrm{s}^{-1}$ might not be detected in the X-ray if absorption were higher than $\sim 10^{23} \mathrm{~cm}^{-2}$. We know that such high absorbing columns are possible because they have been inferred for two detected FU Ori stars: FU Ori and HBC 722. Assuming typical gas-todust ratios for $\mathrm{T}$ Tauri stars, these gas column densities would correspond to $>50 \mathrm{mag}$ of extinction in the $V$-band, which would completely obscure an optical source. Given that both these stars are seen in the optical, X-ray absorption does not necessarily correspond to optical extinction. This makes sense in the standard picture of FU Ori stars where optical emission is dominated by the disk, while X-ray emission may originate closer to the star in regions that become obscured by the inward advance of the disk. On the other hand, the changes in star-disk geometry that occur with an eruption do not necessarily result in high $N_{\mathrm{H}}$, since moderate $N_{\mathrm{H}}$ has been observed for systems like V960 Mon and V1735 Cyg.

Several of these scenarios can be tested observationally through follow-up monitoring of FU Ori stars in the X-ray. For example, if outbursts cause increases in X-ray emission, then $\mathrm{X}$-ray properties should be observed to change during outbursts. On the other hand, if the high X-ray luminosity of a YSO statistically increases the outburst rate, these stars should also appear as high $L_{\mathrm{X}}$ objects before the beginning of their outbursts. So far, no known FU Ori star has been observed with XMM-Newton or Chandra before an eruption. However, with ongoing time-domain surveys searching for FU Ori outbursts, it is increasingly likely that an outburst will be discovered in a field already observed by one of these telescopes. X-ray surveys of nearby star-forming regions $(d<3 \mathrm{kpc})$ have identified tens of thousands of YSOs, and there are likely hundreds of thousands of YSOs projected in 
these fields that have not been detected (Feigelson 2018). If the outburst rate were $\sim 10^{-5} \mathrm{yr}^{-1}$, as suggested by Contreras Peña et al. (2019), at least one outburst is likely in this sample of stars during the next decade, allowing constraints on preoutburst X-ray luminosity to be determined.

We thank the XMM-Newton team for approving Gaia $17 \mathrm{bpi}$ as an unanticipated TOO. We would like to thank Hannah Earnshaw for advice on reducing the XMM-Newton data, Matthew Povich for useful discussions about star-forming regions, and the anonymous referee for suggestions that improved the manuscript.

Facilities: XMM-Newton, Chandra X-ray Observatory.

Software: CIAO (Fruscione et al. 2006), HEASOFT (HEASARC 2014), PIMMS (Mukai 1993), R (R Core Team 2018), SAS (SAS Development Team 2014), survival (Therneau \& Grambsch 2000), TOPCAT (Taylor 2005), XSPEC (Arnaud 1996).

\section{Appendix A X-Ray Properties of Individual FU Ori Stars}

The X-ray properties or upper limits of FU Ori stars included in Table 2 are compiled from a variety of sources described below. The ObsIDs for XMM-Newton and Chandra observations are provided in cases where archival data is reanalyzed. $\mathrm{X}$-ray luminosities used here are adjusted for the X-ray energy band using XSPEC and scaled for assumed distance (Appendix B).

\section{A.1. Detected Sources}

\section{A.1.1. V960 Monocerotis}

V960 Mon was observed shortly after the beginning of its outburst by Chandra (ObsID 17587; PI D. Pooley) and $1.75 \mathrm{yr}$ later by XMM-Newton (ObsID 0781690201; PI M. Güdel). The Chandra data were taken with the ACIS-S3 chip (49 ks) in "very faint" mode. We reduced the data with CIAO (Fruscione et al. 2006), using the standard data reduction procedures to obtain CCD spectra from imaging data. ${ }^{7}$ The XMM-Newton data was taken using all three EPIC instruments (78 ks) using the medium filter. These data were reduced using the same procedure as in Section 2.1. For both the Chandra data and the XMM-Newton data, spectral modeling was performed using XSPEC (Arnaud 1996). The fits to these spectra are shown in Figure 5.

For the Chandra data, we found that a one-temperature absorbed plasma wabs(apec) provided an adequate fit, with $N_{\mathrm{H}}=3.9 \pm 1.3 \times 10^{21} \mathrm{~cm}^{-2}, \quad k T=2.6_{-0.4}^{+0.8} \mathrm{keV}, \quad$ norm $=$ $5.6 \pm 0.8 \times 10^{-5}{ }^{8}$ We fixed the elemental abundances in the apec model to 0.4 times solar values for consistency with the rest of the analysis and because it is difficult to accurately estimate abundances from CCD spectra. The data were fit to the unbinned spectrum using the $C$-statistic (Cash 1979) option in XSPEC, using the steppar tool to estimate $1 \sigma$ confidence intervals. The parameters obtained this way also provided a good fit to the binned data shown in Figure 5, with a $\chi^{2}$ value of 8.6 on 12 degrees of freedom. The model fit yields an absorbed X-ray flux of $4.2 \times 10^{-14} \mathrm{erg} \mathrm{s}^{-1} \mathrm{~cm}^{-2}$ and an

\footnotetext{
7 http://cxc.harvard.edu/ciao/guides/

8 In the apec model, the the normalization parameter is related to the emission measure, EM, by norm $=\frac{10^{-14} \mathrm{EM}}{4 \pi D^{2}}$.
}

absorption-corrected X-ray luminosity of $L_{\mathrm{X}}=9.4 \times$ $10^{30} \mathrm{erg} \mathrm{s}^{-1}$ in the $0.5-8.0 \mathrm{keV}$ band. Additional temperature components did not significantly improve the fit.

At a separation of 5!"6 from V960 Mon, a fainter X-ray source (6:59:31.7-4:05:22) can be seen in the Chandra image that is sufficiently near to be unresolved by XMM-Newton. We fit this source with $N_{\mathrm{H}}=1.9 \times 10^{22} \mathrm{~cm}^{-2}, k T=1.5 \mathrm{keV}$, and norm $=2.5 \times 10^{-5}$, yielding an observed flux of $F_{\mathrm{X}}=6.0 \times$ $10^{-15} \mathrm{erg} \mathrm{s}^{-1} \mathrm{~cm}^{-2}$. For the XMM-Newton analysis, this component is included in all models but has a relatively minor effect on the results.

To model the XMM-Newton spectrum of V960 Mon, we fit both one-temperature wabs(apec) and two-temperature models wabs (apec+apec) for the main source, along with an additional component for the neighboring source as described above. The data were simultaneously fit to both the pn and MOS detector spectra using XPEC. The one-temperature model for V960 Mon favored a temperature of $k T \approx 0.7 \mathrm{keV}$; however, it resulted in a moderately poor fit with a $\chi^{2}$ value of 75 on 55 degrees of freedom. For the two-temperature model, $N_{\mathrm{H}}=9.0_{-2.4}^{+1.5} \times 10^{21}$ $\mathrm{cm}^{-2}, k T_{1}=0.54_{-0.18}^{+0.09} \mathrm{keV}$, norm $_{1}=9.9_{-6.0}^{+6.1} \times 10^{-5}, k T_{2}=$ $2.6_{-0.6}^{+2.9} \mathrm{keV}$, and norm $2=2.4_{-1.2}^{+1.3} \times 10^{-5}$, yielding an absorbed $\mathrm{X}$-ray flux of $F_{\mathrm{X}}=2.7 \times 10^{-14} \mathrm{erg} \mathrm{s}^{-1} \mathrm{~cm}^{-2}$ and an absorptioncorrected X-ray luminosity of $L_{\mathrm{X}}=2.3 \times 10^{31} \mathrm{erg} \mathrm{s}^{-1}$ in the $0.5-8.0 \mathrm{keV}$ band. The two-temperature model provides an adequate fit with a $\chi^{2}$ value of 59 on 53 degrees of freedom.

Between the first and second epochs, several changes in the $\mathrm{X}$-ray spectrum have occurred. The overall absorption has increased by a factor of more than two. The plasma temperature at the first epoch is $2.6 \mathrm{keV}$, while at the second epoch, one of the plasma components still has a temperature of $2.6 \mathrm{keV}$, albeit less constrained by the model, with a decrease in emission measure by a factor of $\sim 2$. This suggests that the magnetic activity that produced the hotter component of the corona of V960 Mon may have persisted between the observations, while possibly decreasing in strength. However, in the second epoch, the analysis strongly indicates the presence of a new, cooler temperature component of $\sim 0.6 \mathrm{keV}$. The existence of this component is supported by both one- and two-temperature fits. Both 0.6 and $2.6 \mathrm{keV}$ components are sufficiently hot that they most likely result from magnetic-reconnection heating of a corona.

\section{A.1.2. Z CMa}

Z CMa was observed in 2003 and 2008 by Chandra (Stelzer et al. 2006, 2009). The Z CMa system contains a Herbig AeBe star and an FU Ori star, with the former contributing little to no X-ray flux (Stelzer et al. 2009). Stelzer et al. (2009) state that both epochs are consistent with the same absorbed two-temperature spectral model and report an observed flux of $\sim 1.6 \times$ $10^{-14} \mathrm{erg} \mathrm{s}^{-1} \mathrm{~cm}^{-2}$. Based on their (loosely constrained) best-fit model to the spectrum, we used XSPEC to calculate an absorption-corrected X-ray luminosity in the $0.5-8.0 \mathrm{keV}$ band of $L_{\mathrm{X}}=1.1 \times 10^{31} \mathrm{erg} \mathrm{s}^{-1}$. For the $L_{\mathrm{X}}$ value tabulated in Table 2, we do not add in X-ray emission from a nearby source that Stelzer et al. (2009) attribute to a jet from Z CMa.

\section{A.1.3. $H B C 722$}

X-ray properties of HBC 722 for the second and third epochs in Table 2 are based on fits by Liebhart et al. (2014) to XMM-Newton and Chandra (unbinned) data. 

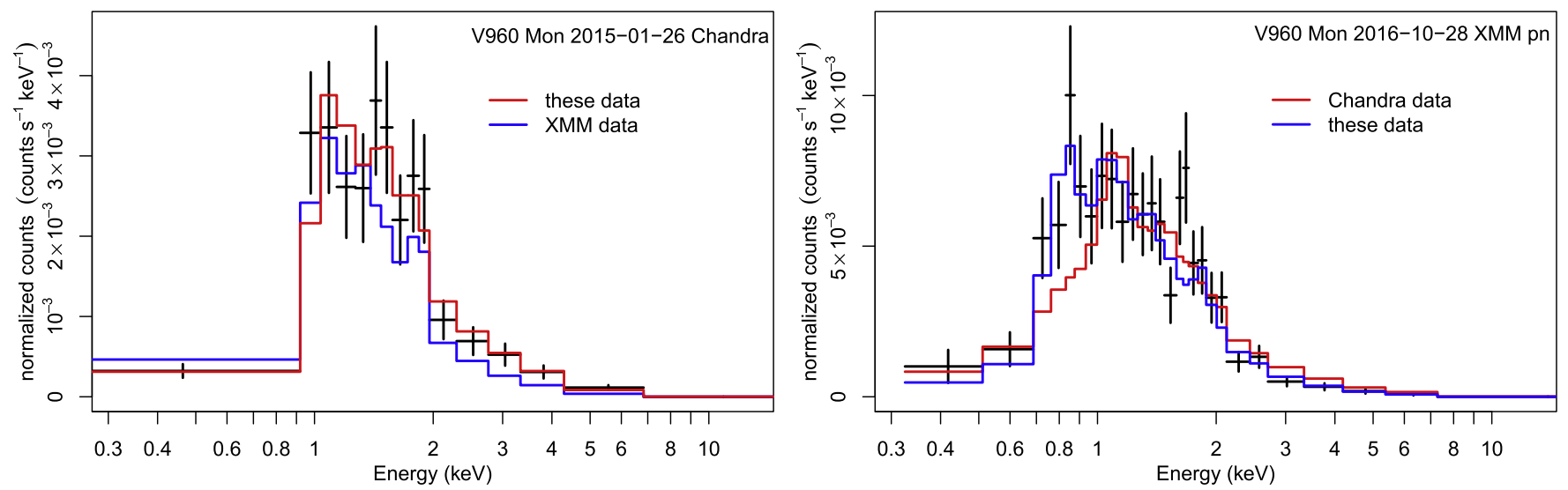

Figure 5. Absorbed plasma models fit to two epochs of X-ray data for V960 Mon observed by Chandra/ACIS-S (left panel) and XMM-Newton/EPIC-pn (right panel). Binned data are shown in black, and the model fits are shown in red (Chandra) and blue (XMM-Newton). The best-fit parameters indicate that the source is more highly absorbed in the second epoch and that a new $\sim 0.6 \mathrm{keV}$ component has appeared in addition to a $\sim 2.6 \mathrm{keV}$ component. As these changes are difficult to perceive in observed spectra, which are dominated by differences in detector response, we show fits to both epochs overlaid on each plot.

Table 3

Upper Limits on X-Ray Fluxes

\begin{tabular}{|c|c|c|c|c|c|c|c|c|}
\hline \multirow{2}{*}{ Object } & \multirow{2}{*}{ ObsID } & \multirow{2}{*}{ PI } & \multirow{2}{*}{ Detector } & \multicolumn{5}{|c|}{ Upper Limit $\left(10^{-15} \mathrm{erg} \mathrm{s}^{-1} \mathrm{~cm}^{-2}\right)$} \\
\hline & & & & Band 1 & Band 2 & Band 3 & Band 4 & Band 5 \\
\hline V883 Orionis & 0205150401 & S. Skinner & pn & 2.66 & 2.97 & 4.34 & 16.34 & 83.92 \\
\hline V2775 Orionis & 0503560301 & S. Wolk & MOS1 & 1.75 & 6.76 & 12.64 & 14.19 & 48.54 \\
\hline V900 Monocerotis & 0783890101 & D. Pizzocaro & pn & 0.31 & 0.35 & 0.74 & 2.77 & 18.7 \\
\hline V1515 Cygni & 0402840101 & S. Skinner & pn & 0.88 & 1.34 & 1.41 & 4.26 & 21.15 \\
\hline HBC 722 & 0656780701 & N. Schartel & pn & 1.43 & 4.04 & 13.87 & 25.06 & 23.08 \\
\hline V1057 Cygni & 0302640201 & S. Skinner & pn & 1.16 & 1.55 & 1.82 & 6.16 & 27.49 \\
\hline V2495 Cygni & 0691580101 & S. Wolk & pn & 0.57 & 0.64 & 1.02 & 3.69 & 20.25 \\
\hline$\ldots$ & 0720890101 & S. Wolk & pn & 0.71 & 0.66 & 1.19 & 4.38 & 34.58 \\
\hline V733 Cephei & $9919,10811,10812$ & T. Allen & ACIS-I & $\cdots$ & $\cdots$ & $\cdots$ & $\cdots$ & $\cdots$ \\
\hline$\cdots$ & 0743980301 & G. Israel & pn & 4.65 & 4.81 & 8.69 & 33.04 & 225.08 \\
\hline IRAS $05450+0019$ & 0201530101 & S. Skinner & pn & 1.13 & 0.99 & 1.79 & 5.95 & 45.69 \\
\hline
\end{tabular}

\section{A.1.4. V1735 Cygni}

X-ray properties of V1735 Cyg are obtained from the model fit by Skinner et al. (2009) to XMM-Newton data.

\section{A.1.5. FU Orionis}

X-ray properties for FU Ori are based on XMM-Newton and Chandra observations by Skinner et al. (2006, 2010). Both observations show an unusual double-peaked X-ray spectrum, composed of a hard component that is likely associated with the outbursting star and a soft component that is likely associated with a companion (Skinner et al. 2010). For each epoch, we use XSPEC to estimate the luminosity of the hard component alone in the $0.5-8 \mathrm{keV}$ band based on the twocomponent models from Skinner et al. (2006, 2010). The Chandra observation shows variability that could be a large coronal flare, so we use the XMM-Newton measurement of $L_{\mathrm{X}}$ as the fiducial "quiescent" $X$-ray luminosity of this object in our analysis.

\section{A.1.6. L1551 IRS 5}

L1551 IRS 5 has been observed by XMM-Newton and Chandra multiple times between 2000 and 2009 (Bally et al. 2003; Schneider et al. 2011). Schneider et al. (2011) interpret the detection of a marginally extended X-ray source as emission from a jet. They find two possible fits to the X-ray spectrum, with their favored solution having the parameters $N_{\mathrm{H}}=1.1 \times 10^{22} \mathrm{~cm}^{-2}, \quad k T=0.6 \mathrm{keV}, \quad$ and $\mathrm{EM}=7.9 \times$ $10^{51} \mathrm{~cm}^{-3}$. This model yields $L_{\mathrm{X}}=10^{28.9} \mathrm{erg} \mathrm{s}^{-1}$, which is the lowest $L_{X}$ for a detected FU Ori star in our sample. An alternate solution, which Schneider et al. (2011) argue is less physically plausible, yields an even lower $L_{X}$ value. However, it is possible that we are not seeing X-ray emission from the star due to high absorption.

\section{A.2. Non-detections}

Table 3 lists information about upper limits from nondetections, including the observation that was used, the detector for which upper limits are calculated, and the upper limits on flux in the five XMM-Newton bands. Notes about upper limits for individual sources (including one derived from Chandra data) are given below. Unless otherwise stated, all upper limits were calculated based on data from 3XMM-DR7 with the FLIX tool using an aperture of $30^{\prime \prime}$ and the energy conversion factors from the $3 \mathrm{XMM}-\mathrm{DR} 7$ documentation. ${ }^{9}$

\footnotetext{
9 http://xmmssc.irap.omp.eu/Catalogue/3XMM-DR7/ECF_DR7.txt
} 
To derive the upper limit for the initial XMM-Newton observation of HBC 722, we use a smaller aperture with a radius of $15^{\prime \prime}$ due to crowding by nearby X-ray sources with overlapping point-spread functions. The FLIX tool identifies flux in several bands, but it is uncertain whether this flux comes from HBC 722 or from its neighbors. For each band, we report the upper limit as the maximum of (1) the upper limit on flux if no sources is detected or (2) a value three standard deviations greater than the measured flux.

The XMM-Newton observation of V900 Mon is not included in the 3XMM-DR7 catalog, so we follow a data reduction and analysis procedure similar to the one used for Gaia 17bpi in Section 2.1. The observation was made on 2016 October 10 using the EPIC instrument with the thin filter. The filtered event list has an ontime of 79,915 s. Upper limits on flux are calculated using the procedure described earlier, within a $15^{\prime \prime}$ aperture at the location of the star (06:57:22.2 - 08:23:18 ICRS) with the conversion from count rate to flux based on the source ARF. V900 Mon was also observed by Chandra in 2007 (ObsID 7475; PI G. Garmire), which also resulted in a non-detection. Due to uncertainty in the date of the eruption, it is unknown whether the star was in an FU Ori state during this observation, so we do not include it in our analysis.

The source V733 Cep was observed in three observations by Chandra, which were included in the Star Formation in Nearby Clouds (SFiNCs; Getman et al. 2017) catalog. SFiNCs provides deep X-ray source lists for this region generated by stacking the all three observations and using sensitive source extraction techniques; however, an X-ray counterpart to V733 Cep was not detected. We empirically estimate the completeness limit of the SFiNCs catalog for sources within $5^{\prime}$ of V733 Cep that have a $N_{\mathrm{H}}$ between $10^{21.5}$ and $10^{22} \mathrm{~cm}^{-2}$. The flux distribution for these sources peaks at $F_{\mathrm{X}}=10^{-13.9} \mathrm{erg} \mathrm{s}^{-1} \mathrm{~cm}^{-2}$ in the $0.5-8.0 \mathrm{keV}$ band, which we interpret as the upper limit for V733 Cep. V733 Cep was also included in an XMM-Newton observation (ObsID 0743980301; PI G. Israel), from which we calculated upper limits based on the $p n$ detector. Given that this observation provides much weaker constraints than the stacked Chandra observations, we use the Chandra upper limit for the analysis in this paper.

Upper limits for V1057 Cyg and V1515 Cyg were previously calculated by Skinner et al. (2009) using the same data sets as us. In the former case, we find a similar upper limit, while in the latter case, our upper limit, using a different assumed distance and spectral model, is lower.

\section{Appendix B Distances to FU Ori Stars}

The Gaia DR2 catalog (Gaia Collaboration et al. 2016, 2018) provides the opportunity to refine distances to various FU Ori stars. Although a few FU Ori stars have reliable parallax measurements, with small formal uncertainties and renormalized unit weight errors that are less than 1.4 as recommended by the Gaia team, ${ }^{10}$ many of the stars do not. Thus, we derive new Gaia-based distances to the stellar associations that host these objects and assume that the FU Ori stars lie at the same distance.

Our procedure is similar to that described by Hillenbrand et al. (2018); we obtain a catalog of probable association members from the literature, match these stars to Gaia DR2 sources, discard stars with parallaxes that are discrepant by $>3 \sigma$, and find the median parallax of the resulting sample.

\footnotetext{
${ }^{10}$ https://www.cosmos.esa.int/web/gaia/dr2-known-issues
}

Several groups have attempted to estimate the zero-point offsets in the Gaia DR2 parallaxes. For the list of star-forming regions below, we report median parallaxes in the Gaia DR2 system but apply a zero-point correction of 0.0523 mas (Leung \& Bovy 2019) when calculating distances, making them slightly smaller. In all cases, uncertainties on the median parallaxes are dominated by the $\sim 0.04$ mas spatially correlated systematic uncertainty reported by the Gaia Collaboration et al. (2018).

Gaia $17 \mathrm{bpi}$ is a probable member of the G53.2 region. The median parallax to probable members of this region is found to be 0.79 mas (Hillenbrand et al. 2018), from which we calculate a distance of $1200_{-70}^{+80} \mathrm{pc}$.

V900 Mon, V960 Mon, and Z CMa are probable members of CMa R1. We estimate a median parallax of 0.84 mas for stars in the association based on 88 probable early-type members cataloged by Shevchenko et al. (1999), yielding a distance of $1120 \mathrm{pc}$. This is consistent with the classical calculation of $1150 \mathrm{pc}$ by Claria (1974).

V1515 Cyg is located in NGC 6914, a star-forming region projected on the sky near Cygnus X. V1515 Cyg has a reliable Gaia DR2 parallax of $0.99 \pm 0.03$, from which we obtain a distance of $960 \mathrm{pc}$.

V733 Cep is a probable member of the Cep OB3 association, which has a median parallax of 1.16 (Kuhn et al. 2019), corresponding to a distance of $825 \mathrm{pc}$.

V1057 Cyg and HBC 722 are both probable members of NGC 7000. We find a median parallax of 1.21 mas for member candidates from the catalog of Damiani et al. (2017), corresponding to $795 \mathrm{pc}$.

V1735 Cyg is associated with IC 5146, which has a median parallax of 1.28 (Kuhn et al. 2019), corresponding to a distance of $752 \mathrm{pc}$.

V2494 Cyg and V2495 Cyg are both probable members of Cyg OB7. We find a median parallax of 1.63 mas for member candidates from Herbig \& Dahm (2006), corresponding to $594 \mathrm{pc}$.

V883 Ori, FU Ori, V2775 Ori, and IRAS 05450+0019are located in the Orion complex. V883 Ori is located in the head of the Orion A cloud, south of the Orion Nebula Cluster, in a region where stars have an average parallax of 2.50 mas (Großschedl et al. 2018), corresponding to a distance of $392 \mathrm{pc}$. FU Ori is located in the $\lambda$ Ori region, which has a Gaia-based distance of 404 pc from Kounkel et al. (2018). V2775 Orionis is located in the L1641 region, near the tail of Orion $\mathrm{A}$, in a region with an average parallax of 2.35 mas Großschedl et al. (2018), corresponding to a distance $416 \mathrm{pc}$. IRAS $05450+0019$ is located in NGC 2071. The median parallax is 2.19 mas, based on a sample of YSO candidates from Megeath et al. (2012) that lie within $15^{\prime}$ of IRAS 05450 +0019 , corresponding to a distance of $446 \mathrm{pc}$.

L1551 IRS 5 is a highly absorbed protostar in the Taurus association that is undetectable by Gaia. The distance derived by Luhman (2018) for L1551 is 145 pc. After applying the Leung \& Bovy (2019) zero-point correction, this becomes $144 \mathrm{pc}$.

\section{Appendix C Optical and Ultraviolet Photometry}

Figure 6 shows the SED of Gaia 17bpi during its eruption using photometry from Hillenbrand et al. (2018) with the 


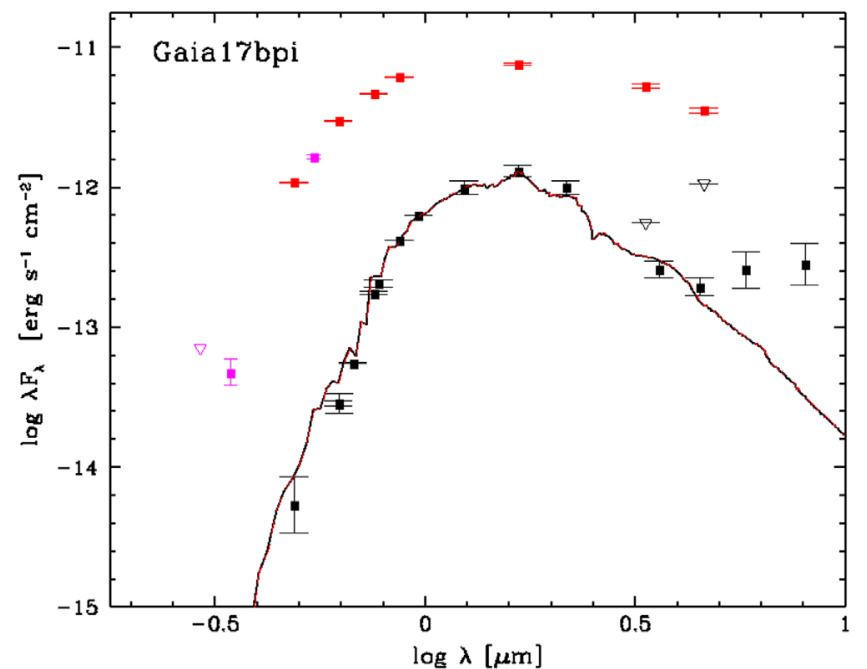

Figure 6. SED of Gaia 17bpi pre-burst (black points and line) and during the outburst (red and magenta points). The magenta points show the new $U$ and $V$ flux measurements and the $U V W 1$ upper limit from the OM. Other points are from Hillenbrand et al. (2018).

$X M M$-Newton OM $U V W 1, U$, and $V$-band photometry newly added here. The source is clearly more luminous in $U$ and $V$ than it would have been in its pre-outburst state (shown for comparison).

\section{ORCID iDs}

Michael A. Kuhn (1) https://orcid.org/0000-0002-0631-7514

\section{References}

Arnaud, K. A. 1996, in ASP Conf. Ser. 101, Astronomical Data Analysis Software and Systems V, ed. G. H. Jacoby \& J. Barnes (San Francisco, CA: ASP), 17

Bae, J., Hartmann, L., Zhu, Z., \& Gammie, C. 2013, ApJ, 764, 141

Bae, J., Hartmann, L., Zhu, Z., \& Nelson, R. P. 2014, ApJ, 795, 61

Bally, J., Feigelson, E., \& Reipurth, B. 2003, ApJ, 584, 843

Baraffe, I., Chabrier, G., Allard, F., \& Hauschildt, P. H. 1998, A\&A, 337, 403

Bonito, R., Orlando, S., Peres, G., Favata, F., \& Rosner, R. 2007, A\&A, 462, 645

Broos, P. S., Townsley, L. K., Feigelson, E. D., et al. 2010, ApJ, 714, 1582

Carrera, F. J., Ebrero, J., Mateos, S., et al. 2007, A\&A, 469, 27

Cash, W. 1979, ApJ, 228, 939

Claria, J. J. 1974, AJ, 79, 1022

Clarke, C., Lodato, G., Melnikov, S. Y., \& Ibrahimov, M. A. 2005, MNRAS, 361, 942

Connelley, M. S., \& Reipurth, B. 2018, ApJ, 861, 145

Contreras Peña, C., Naylor, T., \& Morrell, S. 2019, MNRAS, 486, 4590

Da Rio, N., Robberto, M., Hillenbrand, L. A., Henning, T., \& Stassun, K. G. 2012, ApJ, 748, 14

Damiani, F., Pillitteri, I., \& Prisinzano, L. 2017, A\&A, 602, A115

Feigelson, E. D. 2018, ASSL, 424, 119

Feigelson, E. D., Broos, P., Gaffney, J. A., III, et al. 2002, ApJ, 574, 258

Feigelson, E. D., Getman, K., Townsley, L., et al. 2005, ApJS, 160, 379

Feigelson, E. D., \& Montmerle, T. 1999, ARA\&A, 37, 363

Feigelson, E. D., \& Nelson, P. I. 1985, ApJ, 293, 192

Fruscione, A., McDowell, J. C., Allen, G. E., et al. 2006, Proc. SPIE, 6270 , $62701 \mathrm{~V}$

Gaia Collaboration, Brown, A. G. A., Vallenari, A., et al. 2018, A\&A, 616, A1

Gaia Collaboration, Prusti, T., de Bruijne, J. H. J., et al. 2016, A\&A, 595, A1

Getman, K. V., Broos, P. S., Kuhn, M. A., et al. 2017, ApJS, 229, 28
Getman, K. V., Feigelson, E. D., Broos, P. S., Micela, G., \& Garmire, G. P. 2008, ApJ, 688, 418

Getman, K. V., Flaccomio, E., Broos, P. S., et al. 2005, ApJS, 160, 319

Gramajo, L. V., Rodón, J. A., \& Gómez, M. 2014, AJ, 147, 140

Gregory, S. G., Adams, F. C., \& Davies, C. L. 2016, MNRAS, 457, 3836

Großschedl, J. E., Alves, J., Meingast, S., et al. 2018, A\&A, 619, A106

Güdel, M. 2004, A\&ARv, 12, 71

Harrington, D. P., \& Fleming, T. R. 1982, Biometrika, 69, 553

Hartmann, L., Herczeg, G., \& Calvet, N. 2016, ARA\&A, 54, 135

Hartmann, L., \& Kenyon, S. J. 1996, ARA\&A, 34, 207

HEASARC 2014, HEAsoft: Unified Release of FTOOLS and XANADU v6.25, Astrophysics Source Code Library, ascl:1408.004

Herbig, G. H., \& Dahm, S. E. 2006, AJ, 131, 1530

Hillenbrand, L. A., Contreras Peña, C., Morrell, S., et al. 2018, ApJ, 869,146

Hodgkin, S. T., Wyrzykowski, L., Blagorodnova, N., \& Koposov, S. 2013, RSPTA, 371, 20120239

Ilgner, M., \& Nelson, R. P. 2006, A\&A, 455, 731

Imanishi, K., Koyama, K., \& Tsuboi, Y. 2001, ApJ, 557, 747

Kaplan, E. L., \& Meier, P. 1958, J. Am. Stat. Assoc., 53, 457

Kastner, J. H., Huenemoerder, D. P., Schulz, N. S., Canizares, C. R., \& Weintraub, D. A. 2002, ApJ, 567, 434

Kóspál, Á., Ábrahám, P., Moór, A., et al. 2015, ApJL, 801, L5

Kounkel, M., Covey, K., Suárez, G., et al. 2018, AJ, 156, 84

Kuhn, M. A., Getman, K. V., Feigelson, E. D., et al. 2010, ApJ, 725, 2485

Kuhn, M. A., Getman, K. V., \& Feigelson, E. D. 2015, ApJ, 802, 60

Kuhn, M. A., Hillenbrand, L. A., Sills, A., Feigelson, E. D., \& Getman, K. V. 2019, ApJ, 870, 32

Leung, H. W., \& Bovy, J. 2019, MNRAS, in press

Liebhart, A., Güdel, M., Skinner, S. L., \& Green, J. 2014, A\&A, 570, L11

Luhman, K. L. 2018, AJ, 156, 271

Megeath, S. T., Gutermuth, R., Muzerolle, J., et al. 2012, AJ, 144, 192

Mukai, K. 1993, Legacy, 3, 21

Pecaut, M. J., \& Mamajek, E. E. 2013, ApJS, 208, 9

Pooley, D., Green, J. D., Skinner, S., \& Guedel, M. 2015, ATel, 7025, 1

Preibisch, T., Kim, Y.-C., Favata, F., et al. 2005, ApJS, 160, 401

Prisinzano, L., Micela, G., Flaccomio, E., et al. 2008, ApJ, 677, 401

R Core Team 2018, R: A Language and Environment for Statistical Computing (Vienna: R Foundation for Statistical Computing)

Reipurth, B., \& Aspin, C. 2010, in Evolution of Cosmic Objects through their Physical Activity, ed. H. A. Harutyunian, A. M. Mickaelian, \& Y. Terzian (Yerevan: NAS RA), 19

Rosen, S. R., Webb, N. A., Watson, M. G., et al. 2019, yCat, 9, 54

Ryter, C. E. 1996, Ap\&SS, 236, 285

SAS Development Team 2014, SAS: Science Analysis System for XMMNewton Observatory v17.0.0, Astrophysics Source Code Library, ascl:1404.004

Schmitt, J. H. M. M., Robrade, J., Ness, J.-U., Favata, F., \& Stelzer, B. 2005, A\&A, 432, L35

Schneider, P. C., Günther, H. M., \& Schmitt, J. H. M. M. 2011, A\&A, 530, A123

Shevchenko, V. S., Ezhkova, O. V., Ibrahimov, M. A., van den Ancker, M. E., \& Tjin A Djie, H. R. E. 1999, MNRAS, 310, 210

Shu, F., Najita, J., Ostriker, E., et al. 1994, ApJ, 429, 781

Skinner, S. L., Briggs, K. R., \& Güdel, M. 2006, ApJ, 643, 995

Skinner, S. L., Güdel, M., Briggs, K. R., \& Lamzin, S. A. 2010, ApJ, 722,1654

Skinner, S. L., Sokal, K. R., Güdel, M., \& Briggs, K. R. 2009, ApJ, 696, 766

Stelzer, B., Hubrig, S., Orlando, S., et al. 2009, A\&A, 499, 529

Stelzer, B., Micela, G., Hamaguchi, K., \& Schmitt, J. H. M. M. 2006, A\&A, 457, 223

Taylor, M. B. 2005, in ASP Conf. Ser. 347, Astronomical Data Analysis Software and Systems XIV, ed. P. Shopbell, M. Britton, \& R. Ebert (San Francisco, CA: ASP), 29

Telleschi, A., Güdel, M., Briggs, K. R., Audard, M., \& Palla, F. 2007, A\&A, 468,425

Therneau, T. M., \& Grambsch, P. M. 2000, Modeling Survival Data: Extending the Cox Model (New York: Springer)

Wang, J., Townsley, L. K., Feigelson, E. D., et al. 2008, ApJ, 675, 464

Zhu, Z., Hartmann, L., Gammie, C. F., et al. 2010, ApJ, 713, 1134 